\title{
A Controller Design Researh Based on the Cloud Model
}

\author{
Feng Jie*, Jingfang Wang \\ School of Information Science \& Engineering, Hunan International Economics University, \\ Changsha, China, postcode: 410205 \\ ${ }^{*}$ Corresponding author, e-mail: matlab_bysj@126.com
}

\begin{abstract}
A novel control structure model is proposed based on cloud model for the first time. The structure model is a nonlinear model in nature, and it can be composed of a group of uncertain reasoning rules easily. Nonlinear mapping characteristics of cloud model is analysed in this sudy, and the design method of the intelligent controller is presented based on the structure model, and some simulation examples are showed.
\end{abstract}

Keywords: cloud model, cloud controller, uncertainty reasoning, nonlinear mapping, intelligent control

Copyright $@ 2015$ Institute of Advanced Engineering and Science. All rights reserved.

\section{Introduction}

Both in artificial intelligence, data mining, intelligent control, or in other fields, the primary problem is how to express knowledge, natural language is clearly the best way of expression. Prevalence between qualitative concepts and quantitative data are uncertainty, especially randomness and fuzziness [1-4]. Cloud model is a uncertainty transformation model between qualitative concept and its quantitative data, which are expressed in natural language values, it is mainly reflecting the fuzziness and randomness of things in the objective world or the concept of human knowledge, and the two are fully integrated, the two constitutings is mapped to each other between qualitative and quantitative $[5,6]$. In the process of spatial data mining and knowledge discovery, the cloud model calculations are using both quantitative analysis and processing of data, but also it is full attention to the role of qualitative thinking and description. Cloud model has been used to the excavation of the space generalized knowledge and association rules, the expression of knowledge discovery, continuous data discretization, the uncertainty query of spatial database and uncertainty reasoning, interpretation and recognition of remote sensing, and other field [7-11]. Cloud model is applied to fuzzy reliability analysis of aircraft navigation systems and its fault detection; it can effectively improve reliability analysis of aircraft navigation system [12]. The cloud model is introduced into the concept of the tree, it is facilitated further to enrich the basic connotation of the concept tree, abstract problem is solved in fuzzy attribute domain, the promotion of concepts are achieved, knowledge extraction is made and prediction rule is done [13]. In the field of intelligent control, the cloud model is applied to the controller design method, it is a useful attempt [14, 15], the control of the human experience is convert to the language control rule by the language of atoms and cloud model, a controller was designed for the inverted pendulum system, and it is for effective control [16]. Based on the one-dimensional and two-dimensional cloud model, a hybrid peacekeeping rule reasoning method is proposed [17], it simplifies the complex control rules. According to the characteristics of electro-hydraulic servo variable pitch system, a twodimensional cloud model controller is designed $[18,19]$.

Although the cloud model has been used in intelligent controller design, some good results have been made in these control attempts, but this is only the beginning, the literature in this area is still limited, so there is still a lot of issues worth further study, such as cloud-based subset of the language model and the uncertainty inference rules, their designs impact the control input-output nonlinear mappings, how designs are simplified in cloud model controller structure. For this study, two aspects were studied, and the relevant simulation results are given. 


\section{Cloud Model}

Let $U$ be a common set $U=\{x\}$, it is called on the field. $T$ is a subset of $U$ on the language, $C_{T}(x)$ is a mapping from $U$ to the closed interval $[0,1]$, for any element $x \in U$, there is a stable tendency random number $C_{T}(x), C_{T}(x)$ distribution of $U$ is called the cloud model [5]. In particular, let R1(E1,E2) be normally distributed random function, which E1 is the expected value, E2 is standard deviation, by satisfying the formula:

$$
\begin{aligned}
& x_{i}=R_{1}(E x, E n) \\
& P_{i}=R_{1}(E n, H e) \\
& \mu_{i}=e^{-\frac{1}{2}\left(\frac{x_{i}-E x}{P_{i}}\right)^{2}}
\end{aligned}
$$

The cloud model is constituted by the data $\operatorname{drop}\left(x_{i}, u_{i}\right)(i=1,2, \cdots)$, it is called a onedimensional normal cloud model [7], it is referred to as the one-dimensional normal cloud, data $\left(x_{i}, u_{i}\right)$ on the composition of the cloud model is called a one-dimensional cloud droplets. Where Ex, En, and He are three important figures feature on the composition of the cloud model, they are called the expected value, entropy and hyper entropy, which is denoted by (Ex, En, $\mathrm{He})$.

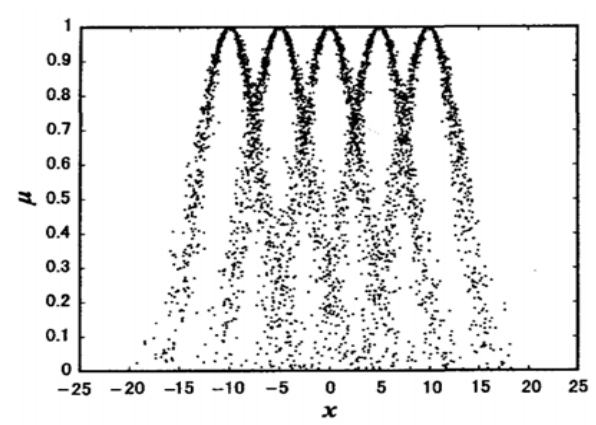

Figure 1. One-dimensional Normal cloud model

Figure 1 is a visual form of five one-dimensional normal cloud model. Clearly, a point map to its distribution value field is one-many relationship, it is not a clear distribution curve. Cloud model overall shape is the most important, distribution characteristic of cloud droplet reflects the fuzziness and randomness of the language concept.

The general composition of the cloud model inference rules contains the two parts of the rule antecedent (conditions) and the rule after pieces (rules knowledge). The known cloud model is (Ex, En, He), if there are particular input $x=x 0$ condition, it is known as cloud model with $X$ condition, it is referred to as $C_{x}$; if a specific condition is $x=C_{T}(x)=C_{T}\left(x_{0}\right)$, it is called cloud model with $Y$ condition $[8,9]$, it is denoted $\mathrm{CG}_{\mathrm{y}}$.

$X$ condition cloud model:

$$
\begin{aligned}
& P_{i}=R_{1}(E n, H e) \\
& \mu_{i}=e^{-\frac{1}{2}\left(\frac{x_{i}-E x}{P_{i}}\right)^{2}}
\end{aligned}
$$

Y condition cloud model:

$$
P_{i}=R_{1}(E n, H e)
$$




$$
y_{i}=E y \pm \sqrt{-2 \ln (\mu)} \bullet P_{i}
$$

Where: in the formula (4), (5), the Ex, En, He is the cloud model digital features (Ex, En, He) of the rule antecedent; the Ey, En, He cloud model digital features after rule (Ey, En, He) in the formula (6), (7).

Figure 2 shows the uncertainty reasoning of the one-dimensional cloud model rules, it is considering only one inference rule. When a particular input $x$ repeatedly stimulate $C G_{x}, C_{x}$ randomly generates a set of $\mu_{i}$ value. These values reflect the intensity of the corresponding activation of qualitative rules, which group $\mu_{i}$ in turn stimulates $\mathrm{CG}_{\mathrm{y}}$, these quantitatively generate a set of random cloud droplets drop $\left(\mathrm{y}_{\mathrm{i}}, \mu_{i}\right)$. In this group of random cloud droplets, the processing methods can be used backward cloud $[10,11]$, the weighted average method can also be used [17], the corresponding quantitative output value y is finally obtained.

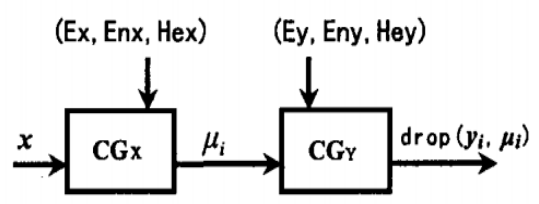

Figure 2. Single Rule Reasoning of one-dimensional Cloud Model

\section{The Nonlinear Mapping of the CIOUD MODel}

Considering the reasoning process and reasoning structure of the one-dimensional cloud model: if $A_{i}$ then $B_{i}, i=1 \sim N$. The domain of cloud model $A_{i}$ is $U[-1,1]$, the domain of cloud model collection $B_{i}$ is $V[-1,1]$, cloud model set is defined as follows, $N=5$.

$$
\begin{array}{ll}
\mathrm{A}_{1}=(-1,0.3,0.01), & \mathrm{A}_{2}=(-0.5,0.3,0.01) \\
\mathrm{A}_{3}=(0,0.3,0.01), & \mathrm{A}_{4}=(0.5,0.3,0.01) \\
\mathrm{A} 5=(1,0.3,0.01), & \mathrm{B} 1=(-1,0.3,0.01) \\
\mathrm{B} 2=(-0.5,0.3,0.01), & \mathrm{B} 3=(0,0.3,0.01) \\
\mathrm{B} 4=(0.5,0.3,0.01), & \mathrm{B}_{5}=(1,0.3,0.01)
\end{array}
$$

According to the above conditions cloud $X, Y$ conditions cloud and quantitative output algorithm of the weighted average, an input $x$ is known, it is belong to $U$, the corresponding output $y$ can be calculated, $y$ is belong to the $V$ part. When $x$ is any change in the $U$, the corresponding output $y$ is the curve 1 in Figure 3 . Wherein the abscissa represents $x$, the ordinate represents $y$. Obviously, this is close to a clear mapping lines, it is similar to the proportional control relationship.

In addition to the one-dimensional cloud model can achieve linear mappings, it can also easily to achieve nonlinear relationship, the cloud model set (8) was revised to (9), taking $N=4$, nonlinear relationship is the curve 2 in Figure 3, apparently, it is similar to the nonlinear saturation characteristics.

$$
\begin{array}{ll}
\mathrm{A} 1=(-1,0.3,0.01), & \mathrm{A} 2=(0,0.3,0.01) \\
\text { A3 }=(0.5,0.3,0.01), & \mathrm{A} 4=(1,0.3,0.01) \\
\text { B1 }=(-1,0.3,0.01), & \mathrm{B} 2=(1,0.3,0.01) \\
\text { B3 }=(1,0.3,0.01), & \mathrm{B}_{4}=(1,0.3,0.01)
\end{array}
$$

The graph 3 in Figura 3 is for cloud model of a formula (10), $N=5$, the cloud model of inference rules is nonlinear relationship. Maintain constant $\mathrm{N}=5$, the cloud model was revised to (11), the different nonlinear mapping curve 4 is obtained, it is shown in Figure 3. 

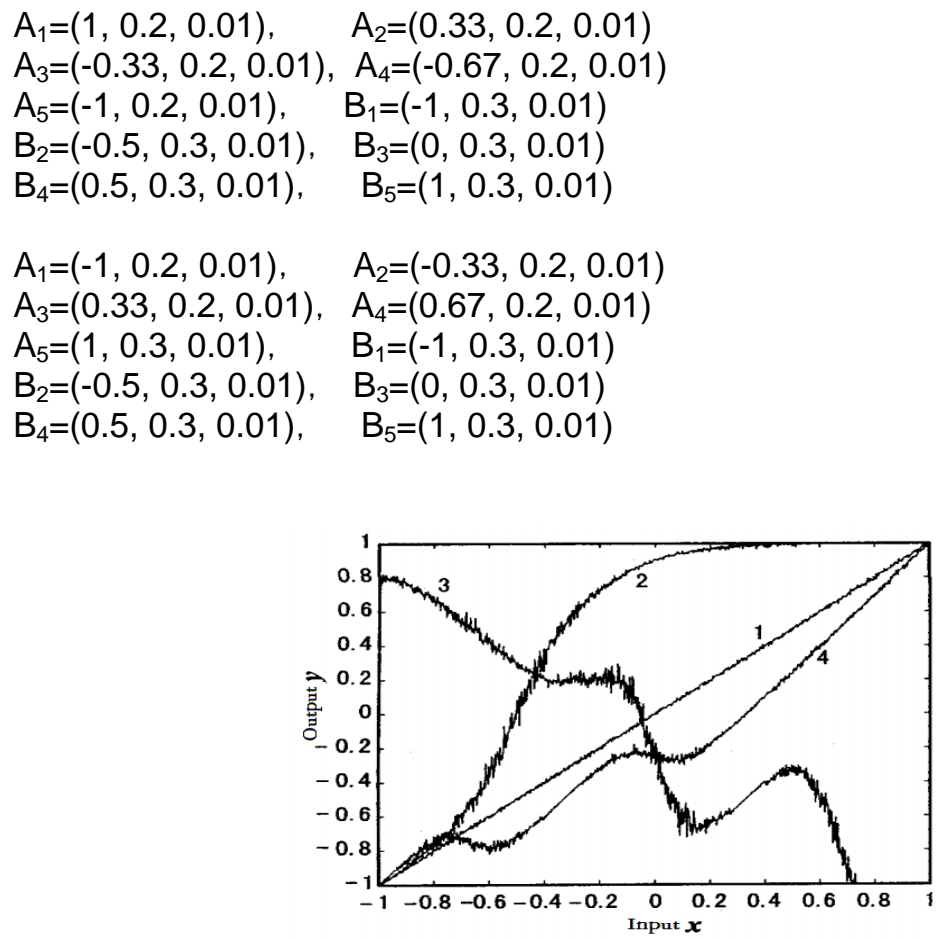

Figure 3. Nonlinear Mapping of the Cloud Model

By this one-dimensional cloud model input and output mapping, the linear mapping function can be achieved either, the certain nonlinear mapping function can also be achieved, which only modify the definition of a limited set of cloud model, or modify several pieces of reasoning rules in the rule base. Accordingly the design, one-dimensional cloud model controller has strong flexibility, the linear controller can be designed, or nonlinear controller design for different controlled objects, in addition, when considering online adjustment rules, the dynamic switching control can be achieved between linear control and nonlinear control.

\section{Design of the Cloud Model Controller}

Essentially, input-output control is realized by the controller, it is actually a mapping relationship, i.e., it is the mapping from the deviation input of the control output. A onedimensional cloud model mapping is considered, it is shown in Figure 4, the map consists of two parts: the multi inference rules of one-dimensional cloud model section and the weighted average processing section. Multi-part rule reasoning actually consists of more than onedimensional single rule-based reasoning structure, the number of rules in the rule base is $N$, where the input $x$ stimulates different parts of the rule antecedent CGA1 $\sim$ CGAN, ${ }^{\mu}$ different values are generated, then it is processed by post pieces rules CGU1 CGUN rules, resulting

in a large number of cloud droplets drop (yNjk, ${ }^{\mu}{ }_{N j}$ ). After the weighted average cloud droplets are treatment, and ultimately the quantitative output value $y$ is obtained, it is the input $x$ corresponding value.

Set deviation $e \in E=[-1,1]$, the deviation integral value ei $\in \mathbb{E I}=[-I, 1]$, the deviation change rate ec $\in \mathrm{EC}=[-1,1]$, quantitative input e, ei and ec is respectively processed by each one-dimensional cloud model mapping, the final output of the three control components is separately with uP, ul and uD. In essence, in the physical sense control, the control of these three components are similar to conventional PID controllers with the functions of the three control components, but also it has a different nature. Set uP $\in$ UP $=[-1,1]$, ul $\in \mathrm{UI}=[-1,1]$ and $\mathrm{uD} \in \mathrm{UD}=[-1,1]$. Without loss of generality, here-100\% is represented by $-1,+100 \%$ is indicated by +1 . For simplicity, e to uP mapping is called the P-type cloud model controller; ei to ul mapping is said for I-type cloud model controller; ec mapping to UD is said for D-cloud model

TELKOMNIKA Vol. 16, No. 3, December $2015: 531-538$ 
controller. P-type, I-type and D-type cloud model controller are essentially belong to the category of one-dimensional cloud model, the difference is that the meaning of input parameters differ: In P-type cloud model controller, the deviation is directly made as the the mapping input of one-dimensional cloud model; in I-type cloud model controller, bias integral value is made as one-dimensional cloud model mapping input; in the D-type cloud model controller, the change rate of the deviation is made as one-dimensional cloud model mapping input.

In the domain of $\mathrm{E}, \mathrm{El}$ and $\mathrm{EC}$, their cloud models were defined:

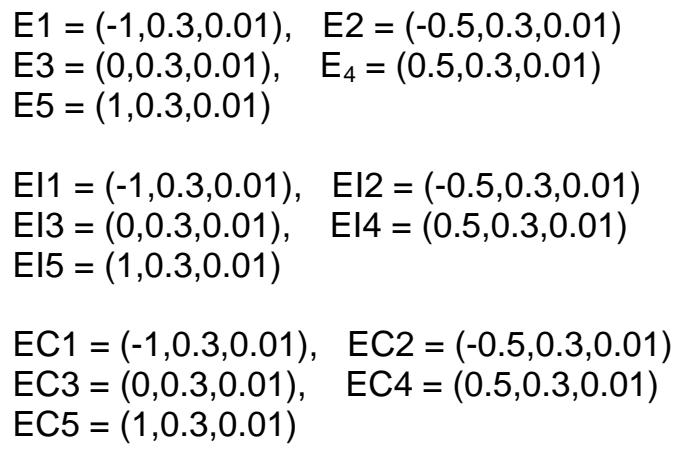

In the domain of UP, UI and UD, the same cloud models are defined:
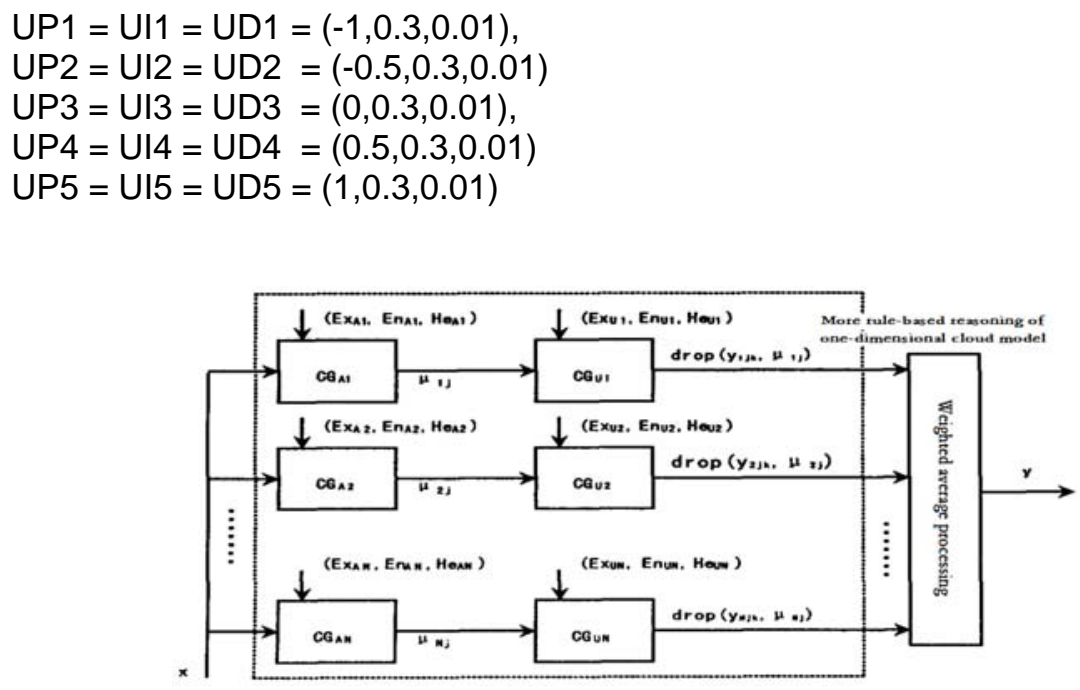

Figure 4. The Mapping Processor of One-Dimensional Cloud Model

P-type cloud model control rules:

If $\mathrm{E}=\mathrm{E} 1$, then UP=UP1

If $E=E 2$, then UP=UP2

If $E=E 3$, then UP=UP3

If $E=E 4$, then UP $=U P 4$

If $E=E 5$, then UP=UP5

Similarly, I-type and D-type cloud model control rules are as follows:

If $\mathrm{EI}=\mathrm{EI} 1$, then $\mathrm{UI}=\mathrm{UI} 1$

If $\mathrm{EI}=\mathrm{EI} 2$, then $\mathrm{UI}=\mathrm{UI} 2$

If $\mathrm{EI}=\mathrm{EI} 3$, then $\mathrm{UI}=\mathrm{UI} 3$

If $\mathrm{EI}=\mathrm{EI} 4$, then $\mathrm{UI}=\mathrm{UI} 4$

If $\mathrm{EI}=\mathrm{EI}$, then $\mathrm{U}_{1}=\mathrm{U}_{15}$ 
If $E C=E C 1$, then UD=UD1

If $E C=E C 2$, then UD=UD2

If $E C=E C 3$, then UD=UD3

If $\mathrm{EC}=\mathrm{EC} 4$, then UD=UD4

If $\mathrm{EC}=\mathrm{EC} 5$, then UD=UD5

\section{Simulation Results and Analysis}

First, the cloud model controller and conventional PID controller are considered in the same controlled object, their control performances are researched in different time lag situations. Controlled object is in formula (19).

Plant 0: $G(s)=\frac{s+2}{s^{3}+22 s^{2}-13 s+43}$

Cloud model controller consists of three separate one-dimensional cloud model controllers: P-type cloud model controller, I-type cloud model controller and D-type cloud model controller, which implement P (proportional) control, I (Integral) control and D (differential) control function, the corresponding magnification is 85,30 and 65 , respectively, and then As the sum of the three control components are as the control amount of the controlled object. Conventional PID three parameters were also taken as $K_{P}=85, K_{1}=30, K_{D}=65$.

Figure 5 is results of cloud model controller and PID controllers, they are on the same controlled object Plant 0 , control performance results are under different delay conditions. Thereare the four curves in Figure 5(a): dotted line, dashed, solid and dotted lines, which are respectively the control effects of cloud model controller under delay $0 \mathrm{~s}, 0.029 \mathrm{~s}, 0.05 \mathrm{~s}$ and $0.1 \mathrm{~s}$ case. There are the two curves in Figure 5(b): dotted and solid lines, which are respectively control effects for the PID controller in the time lag 0s and 0.029s case, and if the stagnations reach to $0.05 \mathrm{~s}$ or $0.1 \mathrm{~s}$, PID control is divergence, the system is not controllable. When the system delays are 0s, the control characteristics curves of cloud model controller and PID controller are almost identical; when the time lag reaches $0.029 \mathrm{~s}$, conventional PID control is critical oscillation state, an enlarged view is seen in Figure $5(\mathrm{~b})$; when delays reach $0.05 \mathrm{~s}$, conventional PID controller has stalled, and P + I + D-type cloud model controller has not spread when the time lag reaches $0.1 \mathrm{~s}$, and the output control is between 0.8-1.15.

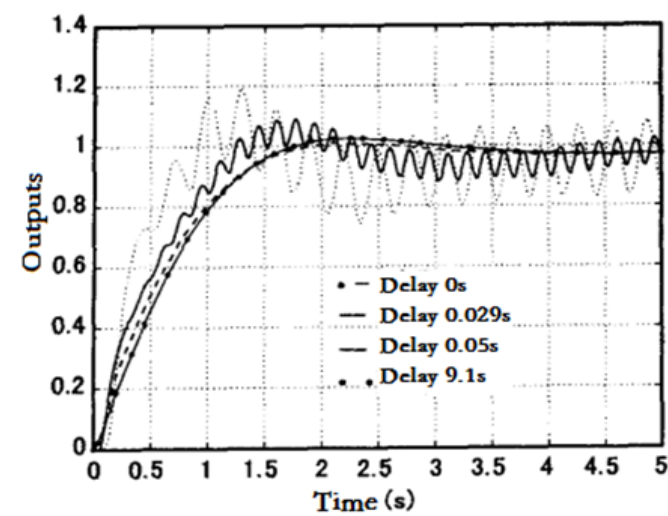

(a) Cloud Model Control Effect

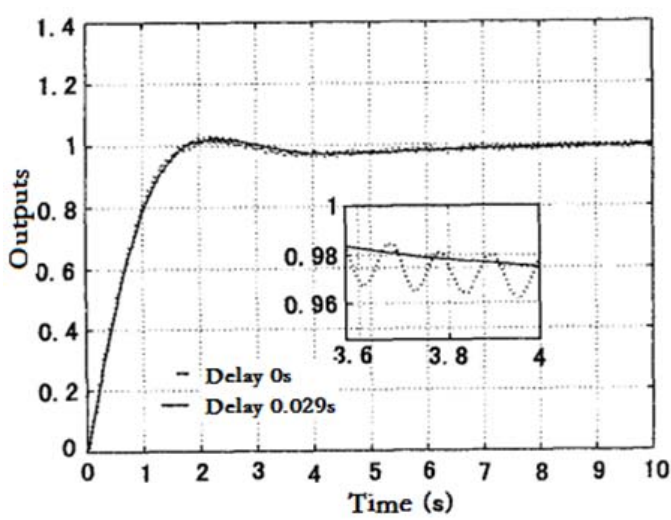

(b) PID Control Effect

Figure 5. Control performance comparison with different delays

The cases are considered when the controlled object changes, three different controlled objects are set as follows:

TELKOMNIKA Vol. 16, No. 3, December 2015 : 531 - 538 
Plant 1: $G(s)=\frac{s+2.3}{s^{3}+1.2 s^{2}+0.4}$

Plant 2: $G(s)=\frac{1}{s^{2}-2 s+3}$

Plant 3: $G(s)=\frac{s-2}{s^{3}+12 s^{2}+37 s+52}$

When the controlled object is changed, the parameters and structure of the two controllers are kept constant, the system Delays are set to 0s. Figure 6(a) shows the control effect of cloud model controller, wherein the object control curve 3 (solid line) is magnified to $10^{2}$ times. Figure 6(b) shows the control effect of the PID controller, wherein the object control curve 3 (solid line) is magnified to $10^{6}$ times. The simulation results are shown in Figure 5 and 6 , it is clear that robust cloud model controller is better than conventional PID controller.

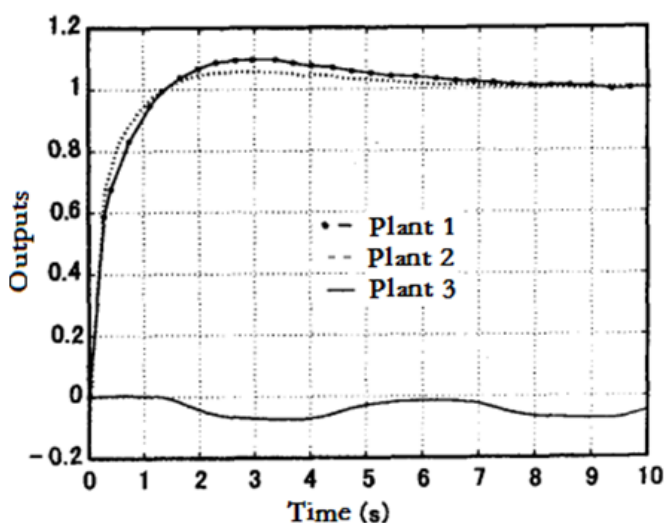

(a) Cloud Model Control Effect

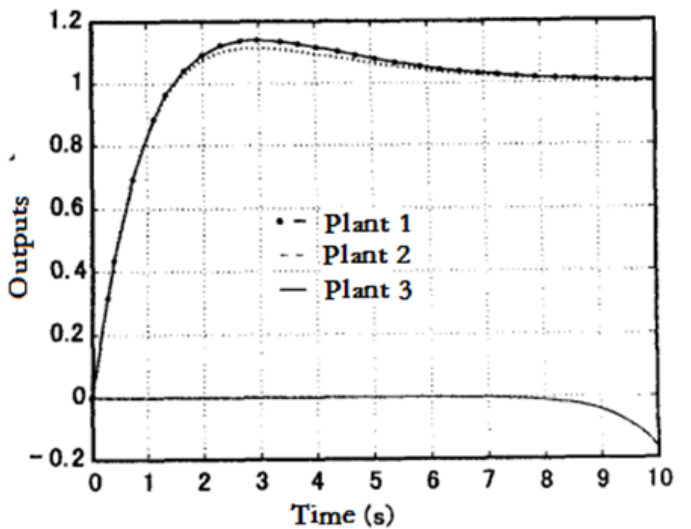

(b) PID Control Effect

Figure 6. Control performance comparison of the different controlled object

\section{Conclusion}

The control method of cloud model is proposed in this study, it is not required to give a precise mathematical model of controlled object, it is based solely on a person's feelings and logic, human qualitative control experience is expressed in natural language, these are converted to language control rules by the cloud model reactor, this method can well realized from quantitative to qualitative, and then it realizes mapping from qualitative to quantitative controls. This method is based on the cloud model controller design, control strategy is clear and intuitive, reasoning is simple, just the digital characteristic parameters and control rules are slightly modified, the different control maps can be achieved. The simulation results show that the design of the controller is successful, robust is strong, there is good application value.

\section{References}

[1] Li deyi, Gan wenyan, Liu luyin. Artificial Intelligence and Cognitive Physics. $10^{\text {th }}$ National Annual Conference Proceedings of Chinese Advances in artificial intelligence. Beijing. 2003: 6-15.

[2] LI DY, Liu CY. Study on the universality of the normal cloud model. Engineering Sciences. 2004; 8: 28-34.

[3] LI DY. Uncertainty in knowledge representation. Engineering Sciences. 2000; 10: 73-79.

[4] Zhang Jinchun, HU Guyu. Application of uncertainty reasoning based on cloud model in time series prediction. JOURNAL OF ZHEJIANG UNIVERSITY (SCIENCE). 2003; 4(5).

[5] Li De-yi, Meng Hai-jun, Shi Xue-mei. Membership clouds and cloud generators. The research and Development of Computers. 1995; 6: 15-20. 
[6] Yang Zhi-xiao, Fan Yan-feng. Cloud mapping and membership cloud of mapping. Application Research of Computers. 2012; 29(2).

[7] Li DY, Shi XM, Ward P. Soft inference mechanism based on cloud models. Bonn, Germany. 1996: 3863.

[8] Li Deyi, Han J, Chan E. Knowledge representation and discovery based on linguistic atoms. Singapore. 1997: 89-97.

[9] Li Deyi, Di Kaichang, Li Deren, Shi Xuemei. Mining association rules with linguistic cloud models. Australia. 1998.

[10] WANG Guoyin, XU Changlin, et al. p-order Normal Cloud Model Recursive Definition and Analysis of Bidirectional Cognitive Computing. Chinese Journal of Computers. 2013; 36(11).

[11] DI Kaichang, LI Deyi, Li Deren. Cloud Theory and Its Applications in Spatial Data Mining and Knowledge Discovery. JOURNAL OF IMAGE AND GRAPHICS. 1999; 4(11): 930-935.

[12] Zhang Feizhou, Fan Yuezu, Sun Xianfang. Assessment and Analysis of Fuzzy Reliability Based on Cloud Models in Aircraft Navigation System. Acta Automatica SINICA. 2002; 28(1): 126-130.

[13] YANG Zhaohui, LI Deyi. Planar Model and its Application in Prediction. Chinese Journal of Computers. 1998; 21(11): 961-969.

[14] Li De-yi, Cheng DW, Shi Xue-mei. Uncertainty reasoning based on cloud models in controllers. Journal of Computer Science and Mathematics with Application.1998; 3: 99-123.

[15] Zhang Feizhou, Fan Yuezu, et al. Intelligent Control Based on membership cloud generator. Acta Aeronautica et Astronautica SINICA. 1999; 20(1): 89-92.

[16] Zhang Feizhou, Fan Yuezu, et al. Intelligent Control Inverted Pendulum with Cloud Models. Control Theory \& Applications. 2000; 17(4): 519-523.

[17] Li Zhong, Yang Yidong. Controllable Pitch Propeller Control Based on Qualitative Reasoning of Variant Dimension Cloud Model. Journal of Nanjing University of Aeronautics \& Astronautics. 2003; 35(2): 162-167.

[18] Li zhong, Gao Jian. Study on Two-Dimension Cloud Model Control for Electric-hydraulic Servo Variable Pitch. Journal of System Simulation. 2004; 16(5): 1050-1052.

[19] Zheng Enrang, Hui Lichuan, et al. Intelligent Control Algorithm of Cloud models and Simulation Research of Superheated Steam Temperature Control. Journal of System Simulation. 2007; 19(1). 